\title{
EXTENT OF ANXIETY AND STRESS AMONG HEALTH CARE WORIKERS IN THE STATE OF SIKIKIM
}

KEY WORDS: Anxiety, Stress, Health Care Workers, Sikkim, Covid-19
Dr. Arunima Sengupta Lahiri

Nima Norbu Sherpa*

\section{Tenzing Sangay} Bhutia
Child Psychologist, All India Institute of Medical Sciences Rishikesh, Uttarakhand.

(Student) Amity Institute of Psychology and Allied Sciences, Amity University Kolkata. *Corresponding Author

Research Officer, Royal Melbourne Institute of Technology, Melbourne, Australia.

The purpose of this study is to determine the level of anxiety and stress among different groups of Sikkim's health-care workers, based on gender and previous exposure to ICU duties. The current study's sample included $152 \mathrm{HCWs}$ who were chosen via stratified random sampling. The anxiety and stress dimension of the Depression Anxiety and Stress Scale (DASS-21) was used to assess anxiety and stress. Based on gender and previous exposure to ICU duty, different groups of health care workers were found to have varying levels of anxiety and stress.

\section{INTRODUCTION}

Many health-care workers (HCWs) have worked extraordinarily long hours in high-pressure conditions throughout the COVID19 pandemic. They may also have been exposed to trauma and/or faced moral difficulties as a result of challenges in providing high-quality treatment, which might be related to a lack of experience or equipment, or a shortage of personnel numbers. HCWs who care for COVID-19 patients are at an elevated risk of infection and, as a result, face the danger of exposing their families, hence, many of them are experiencing anxiety and stress on a constant loop. According to the APA website, anxiety is a feeling of tension, anxious thoughts, and bodily changes such as elevated blood pressure. Anxiety disorders are characterized by repeated intrusive thoughts or concerns. Physical symptoms may include sweating, trembling, disorientation, or a fast pulse. According to 'Frontiers in Psychology', during COVID-19 lockdown, several Health Care personnel experienced moderate to severe anxiety, stress, and despair. Yongyan Deng, et.al (2021) found that healthcare workers have a higher risk for anxiety than the general population. Thakur, et.al (2021) found that anxiety, stress, depression, and insomnia were found highest in frontline healthcare workers than in general healthcare workers. Weibelzahl, et.al (2021) found that job insecurity and infection of family members was the single most important factor for symptoms like depression and anxiety. Lasalvia, et.al (2021) found that the healthcare workers suffered from post-traumatic stress, anxiety and depression due to this pandemic. Gabriele, et.al (2021) found that the Covid-19 pandemic has a serious impact on healthcare workers' mental health. They are suffering from anxiety, depression, insomnia, PTSD, suicidal ideas, sleep disorders, drugs, and alcohol consumption. Kisely et al (2020) identified a significant correlation of the psychological health of the healthcare workers during emerging viruses like SARS, MERS with anxiety, trauma, stress, depression and sleep problems. Li, et.al (2020) found in their study that medical staff has a high rate of insomnia and stress. Limited number of Indian researches on anxiety and stress have been done in the north-east India. The aim of the study is to evaluate the same in the health care workers of Sikkim and compare them among gender and previous exposure to ICU duty. Keeping in mind the socio-economic-cultural differences among various states of India and filling the dearth of available studies, the present work was conducted.

\section{Objectives}

To study the differential extent of anxiety and stress of different groups based on gender and previous exposure among HCW.

\section{Hypothesis}

Different groups based on gender and previous exposure will have different extent of anxiety and stress among HCW.

\section{Method \\ 4.1. Sample}

This sample contained 152 individuals (healthcare workers) from around the state of Sikkim, India, who volunteered for the study. Health Care Workers, which included doctors, nurses, and administrative staff, were the subjects of the study. 76 men and women, respectively took part in the study.

\subsection{Instruments}

1. The Depression, Anxiety, and Stress Scale - 21 Items (DASS21): Lovibond and Lovibond created this scale. Every one of the three DASS-2 1 scales has seven things that are assembled into subscales that have comparable substance. Dysphoria, sadness, debasement of life, self-belittling, absence of interest/support, anhedonia, and idleness are completely surveyed on the downturn scale. Autonomic excitement, skeletal muscle impacts, situational tension, and emotional vibe of restless influence are totally estimated on the nervousness scale. Constant vague excitement levels are touchy to the pressure scale. It assesses anxiety, restless excitement, and being effortlessly disturbed/upset, bad tempered/over-responsive, and fretful. Adding the scores for the significant components yields discouragement, nervousness, and stress scores. The unwavering quality of the DASS-2 1 was shown by Cronbach's upsides of $0.81,0.89$, and 0.78 for the burdensome, nervousness, and stress subscales, individually. It has high discriminative, simultaneous, and focalized legitimacy, just as high inner consistency. It corresponds well with the State-Trait Anxiety Scale and oneself rating misery scale.

2. Personal Data Questionnaire: The researcher created a Personal Data Questionnaire. It contained information regarding each participant's name, age, previous covid duty experience, and gender.

\section{Procedure}

The participants were given the Depression, Anxiety, and Stress Scale i.e.,DASS-21 with sufficient instruction. The information was gathered and scored (anxiety and stress dimension only) using the scoring keys provided. There were four subgroups, and the total of each subgroup was computed, as well as their percentage representation were calculated. Bar graphs were used to display the observed scores. 


\section{Result And Discussion}

With the goal of identifying and comparing the level of anxiety and stress among Sikkim's health-care workers (HCWs) in terms of gender, anxiety, stress and past exposure to ICU duties, the following results were found:-

\section{Table I-Table Of Anxiety}

Showing the comparison of levels of anxiety in male and female healthcare workers into five categories i.e, normal, mild, moderate, severe, extremely severe.

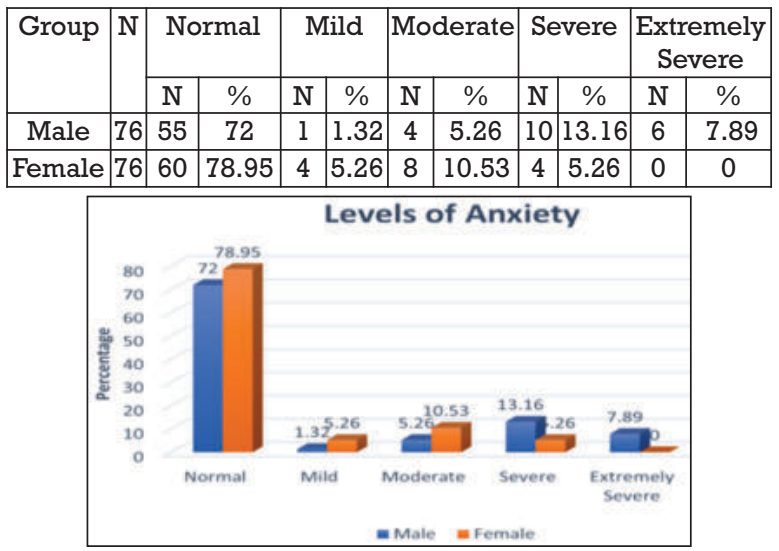

The degrees of anxiety in male and female healthcare workers are categorised into five categories: normal, mild, moderate, severe, and extremely severe.

Table I indicates that $72 \%$ of male HCW suffer from normal anxiety as compared to $78.95 \%$ of female HCW, $5.26 \%$ of male HCW suffer from moderate anxiety as compared to $10.53 \%$ of female HCW. The table also indicates $1.32 \%$ of male HCW compared to $5.26 \%$ of female HCW shows mild anxiety whereas severe anxiety was showed by $13.16 \%$ of male HCW against $5.26 \%$ female HCW. $7.89 \%$ of male HCW showed extremely severe anxiety level against no representation from female HCW.

This suggests that a higher proportion of male HCW suffer from severe and extremely severe anxiety than female HCW, while a higher proportion of female HCW suffer from normal, mild, and moderate anxiety than male HCW.

\section{Table II-Table of Stress}

Showing the comparison of levels of stress in male and female healthcare workers into five categories i.e., normal, mild, moderate, severe, extremely severe.

\begin{tabular}{|c|c|c|c|c|c|c|c|c|c|c|c|}
\hline \multirow[t]{2}{*}{ Group } & \multirow[t]{2}{*}{$\mathrm{N}$} & \multicolumn{2}{|c|}{ Normal } & \multicolumn{2}{|c|}{ Mild } & \multicolumn{2}{|c|}{ Moderate } & \multicolumn{2}{|c|}{ Severe } & \multicolumn{2}{|c|}{\begin{tabular}{|c|} 
Extremely \\
Severe
\end{tabular}} \\
\hline & & $\mathrm{N}$ & $\%$ & $\mathrm{~N}$ & $\%$ & $\mathrm{~N}$ & $\%$ & $\mathrm{~N}$ & $\%$ & $\mathrm{~N}$ & $\%$ \\
\hline Male & 76 & 55 & 72.37 & 2 & 2.63 & 3 & 3.95 & 10 & 13.16 & 6 & 7. \\
\hline emale & 76 & 61 & 80.26 & 3 & 3.95 & 5 & 6.58 & 6 & 7.89 & 1 & \\
\hline
\end{tabular}

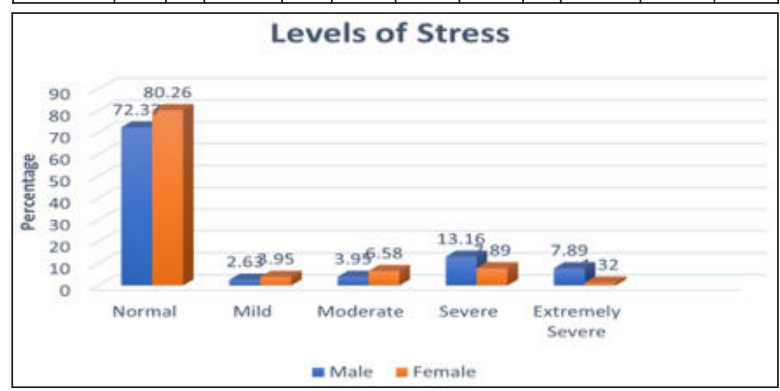

The degrees of stress in male and female healthcare workers are categorised into five categories: normal, mild, moderate, severe, and extremely severe.

Table II indicates that $80.26 \%$ of female HCW had normal level of stress as compared to $72.37 \%$ of male HCW. $2.63 \%$ of male HCW experienced mild stress level against $3.95 \%$ of female HCW. It can be seen from the table that $3.95 \%$ of male and $6.58 \%$ of female HCW suffered from moderate stress and $13.16 \%$ of male HCW experienced severe stress as compared to $7.89 \%$ of female HCW. Extremely severe stress was reported by $7.89 \%$ of male HCW against only $1.32 \%$ female HCW

This suggests that a higher proportion of male HCW suffered from severe and extremely severe stress than female HCW, while a higher proportion of female HCW suffered from normal, mild, and moderate stress than male HCW.

\section{Table III- Table of Anxiety}

Showing the comparison of levels of anxiety in HCW with previous exposure and without previous exposure into five categories i.e., normal, mild, moderate, severe, extremely severe

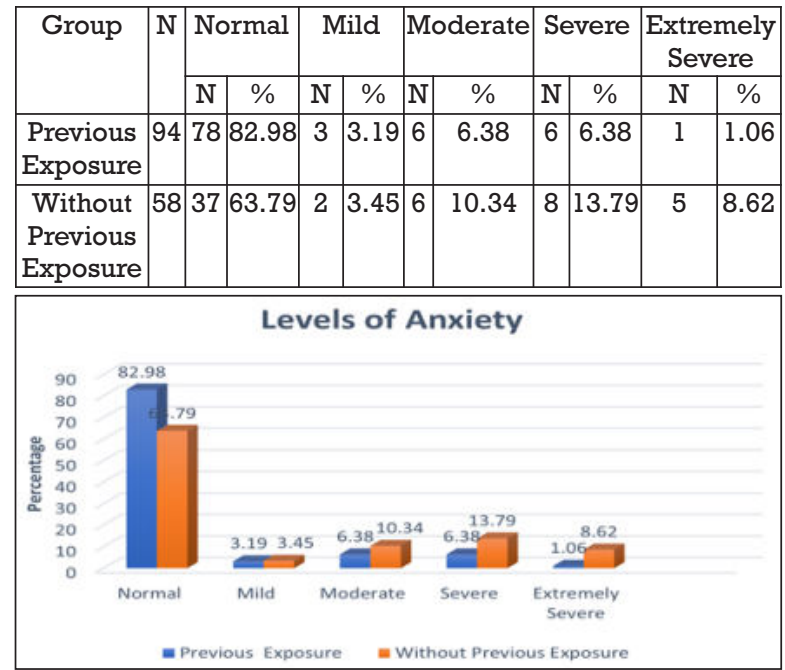

Table III indicates that while $82.98 \%$ of $\mathrm{HCW}$ with previous exposure showed normal level of anxiety, $63.79 \%$ of $\mathrm{HCW}$ without previous exposure experienced the same. $3.19 \%$ of HCW with previous exposure and $3.45 \%$ of HCW without previous exposure had mild anxiety. Moderate anxiety was shown by $6.38 \%$ of $\mathrm{HCW}$ with previous exposure as compared to $10.34 \% \mathrm{HCW}$ without previous exposure. $6.38 \%$ of HCW with previous exposure suffered from severe anxiety whereas $13.79 \%$ of HCW without previous had the same. Extremely severe anxiety was shown by $8.62 \%$ of HCW without previous exposure as compared to $1.06 \%$ of $\mathrm{HCW}$ with previous exposure.

This finding suggests that although a higher number of HCW with previous exposure have normal level of anxiety than those without previous exposure, a greater proportion of HCW without previous exposure suffer from mild, moderate, severe, and extremely severe anxiety as compared to HCW with previous exposure which is aligned with the findings of Neto, et.al (2021) \& Thakur, et. al (2021), who found that depression and anxiety were significant among the healthcare workers during the COVID-19 pandemic, especially those who are working closer to the patient's infected with Covid-19 and anxiety, stress, depression, and insomnia were found highest in frontline healthcare workers than in general healthcare workers.

\section{Table IV-Table of Stress}

Showing the comparison of levels of stress in HCW with previous exposure and without previous exposure into five categories i.e, normal, mild, moderate, severe, extremely severe

\begin{tabular}{|c|c|c|c|c|c|c|c|c|c|c|c|}
\hline \multirow[t]{2}{*}{ Group } & \multirow[t]{2}{*}{$\mathrm{N}$} & \multicolumn{2}{|c|}{ Normal } & \multicolumn{2}{|c|}{ Mild } & \multicolumn{2}{|c|}{ Moderate } & \multicolumn{2}{|c|}{ severe } & \multicolumn{2}{|c|}{$\begin{array}{c}\text { Extremely } \\
\text { Severe }\end{array}$} \\
\hline & & $\mathrm{N}$ & $\%$ & $\mathrm{~N}$ & $\%$ & $\mathrm{~N}$ & $\%$ & $\mathrm{~N}$ & $\%$ & $\mathrm{~N}$ & $\%$ \\
\hline
\end{tabular}




\begin{tabular}{|c|c|c|c|c|c|c|c|c|c|c|c|}
\hline $\begin{array}{c}\text { Previous } \\
\text { Exposure }\end{array}$ & 94 & 77 & 81.91 & 3 & 3.19 & 5 & 5.32 & 7 & 7.45 & 2 & 2.13 \\
\hline $\begin{array}{c}\text { Without } \\
\text { Previous } \\
\text { Exposure }\end{array}$ & 58 & 39 & 67.24 & 2 & 3.45 & 3 & 5.17 & 9 & 15.52 & 5 & 8.62 \\
\hline
\end{tabular}

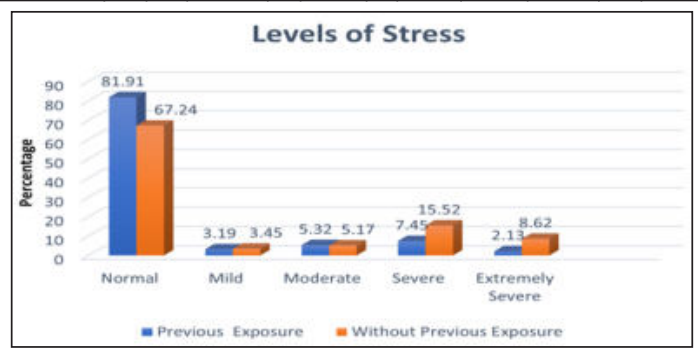

Table IV indicates that $81.91 \%$ of HCW with previous exposure and $67.24 \%$ without previous exposure had normal stress level. The table further indicates that $3.19 \% \mathrm{HCW}$ with previous exposure and $3.45 \%$ of HCW without previous exposure showed mild level of stress. Moderate stress was experienced by $5.32 \%$ of HCW with previous exposure as compared to $5.17 \%$ of $\mathrm{HCW}$ without previous exposure. Severe stress was reported by $15.52 \%$ of HCW without previous exposure and $7.45 \%$ of $\mathrm{HCW}$ with previous exposure. Finally, $2.13 \%$ of HCW with previous exposure suffered from extremely severe stress as compared to $8.62 \%$ of HCW without previous exposure.

This finding suggests that a greater proportion of HCW without previous exposure suffered from mild, severe, and extremely severe anxiety than those with previous exposure, whereas a greater proportion of HCW with previous exposure have a normal and moderate level of anxiety than those without previous exposure. This is in sync with the findings of Lasalvia, et.al (2020), Mustafa, et.al (2020), Shreffler, et.al (2020), Li et al (2020) and Tayyiba, et.al (2020), who all found in their respective studies that most of the healthcare workers were suffering from stress during their duty with Covid infected patients.

\section{Conclusions}

Different groups of health care workers based on gender and previous exposure have different extent of anxiety and stress.

\section{REFERENCES}

1. An, Y., Yang, Y., Wang, A., Li, Y., Zhang, Q., Cheung, T., ... \& Xiang, Y. T. (2020). Prevalence of depression and its impact on quality of life among frontline nurses in emergency departments during the COVID-19 outbreak. Journal of affective disorders, 276, 312-315.

2. da Silva,F.C.T., \& Neto, M.L.R. (2021).Psychiatric symptomatology associated with depression, anxiety, distress, and insomnia in health professionals working in patients affected by COVID-19: a systematic review with metaanalysis. Progress in Neuro-Psychopharmacology and Biological Psychiatry, 104,110057

3. Deng,Y., Chen,Y., \& Zhang, B. (2021). Different prevalence trend of depression and anxiety among healthcare workers and general public before and after the peak of COVID-19 occurred in China: a meta-analysis. Asian journal of psychiatry, 56, 102547.

4. Elamin, M. M., Hamza, S. B., Abdalla, Y. A., Mustafa, A. A. M., Altayeb, M. A., Mohammed, M.A., ... \& Abass, M. F.M. (2020). The psychological impact of the COVID-19 pandemic on health professionals in Sudan 2020. Sudan Journal of Medical Sciences, 15(2), 54-70.

5. Gabriel, K.P., \& Aguinis, H. (2021). How to prevent and combat employee burnout and create healthier workplaces during crises and beyond. Business Horizons.

6. Kisely, S., Warren, N., McMahon, L., Dalais, C., Henry, I., \& Siskind, D. (2020). Occurrence, prevention, and management of the psychological effects of emerging virus outbreaks on healthcare workers: rapid review and metaanalysis. bmj, 369 .

7. Lasalvia, A., Amaddeo, F, Porru, S., Carta, A., Tardivo, S., Bovo, C., \& \& Bonetto, C. (2021). Levels of burn-out among healthcare workers during the COVID-19 pandemic and their associated factors: a cross-sectional study in a tertiary hospital of a highly burdened area of north-east Italy. BMJ open, 11(1), e045127.

8. Lyons, N., Birtus, M., \& Cug, J. (2021). Sustained Psychological Distress, Acute Depression, and Emotional Exhaustion in Frontline Medical Staff and Nurses Working with COVID-19 Patients. Psychosociological Issues in Human Resource Management, 9(1), 99-108.

9. Mahmud, S., Hossain, S., Muyeed, A., Islam, M. M., \& Mohsin, M. (2021). The Global Prevalence of Depression, Anxiety, Stress, and, Insomnia and Its' Changes among Health Professionals during COVID-19 Pandemic: A Rapid Systematic Review and Meta-Analysis. Heliyon, e07393.

10. Sharma, S. K., Mudgal, S. K., Thakur, K., Parihar, A., Chundawat, D. S., \& Joshi, J.
(2021). Anxiety, depression and quality of life (QOL) related to COVID-19 among frontline health care professionals: A multicentric cross-sectional survey.Journal of Family Medicine and Primary Care, 10(3), 1383.

11. Wasim,T.,e Raana, G.,Bushra,N., \& Riaz,A. (2020).Effect of COVID-19 pandemic on mental wellbeing of healthcare workers in tertiary care hospital. Annals of King Edward Medical University,26(Special Issue), 140-144. 